PROCEEDINGS OF THE

AMERICAN MATHEMATICAL SOCIETY

Volume 137, Number 3, March 2009, Pages 971-980

S 0002-9939(08)09544-0

Article electronically published on September 11, 2008

\title{
FOURIER SPECTRUM CHARACTERIZATION OF HARDY SPACES AND APPLICATIONS
}

\author{
TAO QIAN, YUESHENG XU, DUNYAN YAN, LIXIN YAN, AND BO YU
}

(Communicated by N. Tomczak-Jaegermann)

\begin{abstract}
We characterize in terms of Fourier spectrum the boundary values of functions in the complex Hardy spaces $H^{p}\left(\mathbb{C}_{ \pm}\right), 1 \leq p \leq \infty$. As an application we extend the Bedrosian identity, originally stated for square-integrable functions, to the $L^{p}(\mathbb{R})$ cases.
\end{abstract}

\section{INTRODUCTION}

Denote by $H^{p}\left(\mathbb{C}_{+}\right)$and $H^{p}\left(\mathbb{C}_{-}\right)$, or in brief $H_{+}^{p}$ and $H_{-}^{p}$, the complex Hardy spaces in the upper and lower complex plane, $\mathbb{C}_{+}$and $\mathbb{C}_{-}$, respectively. The present paper is devoted to giving a Fourier spectrum characterization for boundary values of the functions in the complex Hardy spaces $H^{p}\left(\mathbb{C}_{ \pm}\right), 1 \leq p \leq \infty$. The spectrum characterization for $H^{2}\left(\mathbb{C}_{ \pm}\right)$is the well known Paley-Wiener Theorem for the functions of positive (negative) spectrum in $L^{2}(\mathbb{R})([17)$, while a generalization of the result to the whole range of $1 \leq p \leq \infty$ has not been systematically addressed. The Fourier spectrum characterization of the boundary values is a further result with important significance to Fourier analysis. We recall that the Fourier transformation for a temperate distribution, $T$, is defined through the relation

$$
\langle\hat{T}, \phi\rangle=\langle T, \hat{\phi}\rangle, \quad \phi \in \mathbb{S}, \quad \text { the Schwartz class, }
$$

which coincides with the traditional definitions of Fourier transformations for functions in $L^{p}(\mathbb{R}), 1 \leq p \leq 2$. Set

$$
\begin{aligned}
\Phi^{+} & :=\left\{\phi \in C_{0}^{\infty}(\mathbb{R}): \operatorname{supp} \phi \subset[0, \infty)\right\} \\
\text { and } \Phi^{-} & :=\left\{\phi \in C_{0}^{\infty}(\mathbb{R}): \operatorname{supp} \phi \subset(-\infty, 0]\right\} .
\end{aligned}
$$

Received by the editors September 20, 2007, and, in revised form, February 24, 2008.

2000 Mathematics Subject Classification. Primary 42A38.

The first author was supported by a research grant from the University of Macau, No. RG079/04-05S/QT/FST, and by the Macao Science and Technology Development Fund $051 / 2005 / \mathrm{A}$.

The second author was supported in part by the U.S. National Science Foundation under grant CCR-0407476, by the Natural Science Foundation of China under grant 10371122, and by the Ministry of Education of the People's Republic of China under the Changjiang Scholar Chair Professorship program.

The third author was supported by the Presidential Foundation of the Graduate School of the Chinese Academy of Sciences (yzjj200505) and NSF of China (10571014 and 10631068).

The fourth author was supported by the NSF of China (Grant No. 10371134/11571082 and 10571014/10631080). 
With this notation, if $f$ is a tempered distribution, then $\operatorname{supp} f \subset[0, \infty)$ if and only if $\langle f, \phi\rangle=0$ for all $\phi \in \Phi^{-}$. Since there is an isometric isomorphism between their boundary values and the functions in the Hardy spaces, we identify the Hardy spaces with their boundary values, denoting the latter by $H_{+}^{p}(\mathbb{R})$ and $H_{-}^{p}(\mathbb{R})$, respectively. The following theorem is established in [15], where for the partial range $p \in[1,2]$ the result was previously known.

Theorem 1.1. If $f$ is the tempered distribution arising from the boundary value of a function in $H^{p}\left(\mathbb{C}_{+}\right), 1 \leq p \leq \infty$, then

$$
\langle\hat{f}, \phi\rangle=0, \quad \text { for all } \phi \in \Phi^{-} .
$$

As the main result of the present paper, we will prove in $\S 2$ the following converse result to Theorem 1.1.

Theorem 1.2. For $1 \leq p \leq \infty$, if $f \in L^{p}(\mathbb{R})$ and $\langle\hat{f}, \phi\rangle=0$ for all $\phi \in \Phi^{-}$, then $f$ is the boundary value of a function in $H^{p}\left(\mathbb{C}_{+}\right)$.

We thus obtain a characterization of the Hardy spaces in terms of the Fourier spectrum of their boundary values. Again, at least for the range $2<p \leq \infty$ the result is new. The proofs for the cases $p=1$ and $p=\infty$ are subtle (see $\S 2$ ). As a direct consequence of Theorems 1.1 and 1.2 we have

Theorem 1.3. $H^{p}(\mathbb{R}), 1 \leq p \leq \infty$, is identical to the Banach subspace of $L^{p}(\mathbb{R})$ of the functions of non-negative spectrum.

Now we justify Hilbert transformations for $L^{p}(\mathbb{R})$ functions, in particular for $p=\infty$. Let $T$ be a real-valued distribution and $u$ its unique harmonic representation in the upper-half complex plane; then $u$ has a harmonic conjugate, $v$, unique up to additive constants. It is proved that $v$ is a harmonic representation of a distribution $S$. Then $S$ is defined to be a Hilbert transform of $T$ that is unique up to additive constants. The above definition is generalized to complex-valued distributions by linearity (see, for instance, [10, 9], 15]; also [1]). If $f \in L^{\infty}(\mathbb{R})$, then the distributional $\mathrm{Hf}$ coincides with the Hilbert transform of $f$ defined by using the modified Hilbert kernel resulting in a BMO function, as shown in [5].

If $f \in L^{p}(\mathbb{R}), 1 \leq p \leq \infty$, then $f$ is the non-tangential boundary value of a function in the Hardy space $H^{p}\left(\mathbb{C}_{+}\right)$if and only if $H f=-i f$, where $H$ is the Hilbert transformation on the line $([15])$. For $p=\infty$ this relation holds in the distribution sense and modulo constants. For $f \in L^{p}(\mathbb{R}), 1 \leq p<\infty$, the usual Hilbert transform $H f$ as a principal-value Cauchy singular integral is selected and thus is uniquely defined. We call such defined $H f$ the conventional branch of the Hilbert transform, or the conventional Hilbert transform of $f \in H^{p}\left(\mathbb{C}_{+}\right)$. Given a function $f \in L^{p}(\mathbb{R}), 1 \leq p<\infty$, using the decomposition $f=f^{+}+f^{-}$where $f^{+}$and $f^{-}$are, respectively, the boundary value of a function in the Hardy space for the upper-half plane and that of one for the lower-half plane, we define $H f=$ $-i\left(f^{+}-f^{-}\right)$to be the conventional Hilbert transform of $f$. This definition coincides with the one defined by using a singular Cauchy integral. The above definition reminds us of the classical Fourier multiplier $-i \operatorname{sgn} \xi$ for Hilbert transformations. Below we proceed to define the conventional branch of the Hilbert transform for a closed subspace of $f \in L^{\infty}$.

Consider the direct sum space $H_{+}^{\infty}+H_{-}^{\infty}$. It is a proper and closed subspace of $L^{\infty}(\mathbb{R})$ (see [6]). A function $f$ in such a space has a unique decomposition 
up to additive constants; that is, if $f=f_{1}^{+}+f_{1}^{-}=f_{2}^{+}+f_{2}^{-}, f_{k}^{ \pm} \in H_{ \pm}^{\infty}$, then $f_{1}^{+}-f_{2}^{+}=f_{2}^{-}-f_{1}^{-}=$constant. By finding the Fourier transform of $f$ in the distribution sense, we can assert the point mass $c_{f} \delta$ contained in $\hat{f}$ that gives rise to the canonical decomposition $f=f^{+}+c_{f}+f^{-}$, where $f^{ \pm} \in H_{ \pm}^{\infty}$, supp $\hat{f^{+}} \subset(0, \infty)$, supp $\hat{f^{-}} \subset(-\infty, 0)$. We define the type-1 conventional Hilbert transform of $f$ to be $H f=-i\left(f^{+}+c_{f}-f^{-}\right)$and the type-2 conventional Hilbert transform of $f$ to be $H f=-i\left(f^{+}-c_{f}-f^{-}\right)$. As an example, taking the simplest case $f=1=1+i 0$, we have $f^{ \pm}=0, c_{f}=1$, and the type-1 conventional Hilbert transform branch $H 1=-i$. Similarly, the type-2 Hilbert transform branch is $H 1=i$.

The above may be summarized into the following:

Definition 1.4. Let $f \in L^{p}(\mathbb{R}), 1 \leq p<\infty$, and let $f=f^{+}+f^{-}$be the unique decomposition of $f$ into the direct sum $H_{+}^{p}(\mathbb{R})+H_{-}^{p}(\mathbb{R})$. The conventional Hilbert transform of $f$ is defined to be $H f=-i\left(f^{+}-f^{-}\right)$.

Definition 1.5. Let $f \in H_{+}^{\infty}(\mathbb{R})+H_{-}^{\infty}(\mathbb{R})$ with the canonical decomposition $f=$ $f^{+}+c_{f}+f^{-}$, where $f^{+} \in H_{+}^{\infty}(\mathbb{R}), f^{-} \in H_{-}^{\infty}(\mathbb{R}), \operatorname{supp} \hat{f^{+}} \subset(0, \infty), \operatorname{supp} \hat{f^{-}} \subset$ $(-\infty, 0)$, and $c_{f}$ is a complex constant. Then the type- 1 conventional Hilbert transform of $f$ is $H f=-i\left(f^{+}+c_{f}-f^{-}\right)$; and the type-2 conventional Hilbert transform of $f$ is $H f=-i\left(f^{+}-c_{f}-f^{-}\right)$.

Based on the above definitions we state the boundary characterization by Hilbert transformations of the spaces $H^{p}\left(\mathbb{C}_{ \pm}\right)$.

Theorem 1.6. For $1 \leq p \leq \infty$, a function $f \in L^{p}(\mathbb{R})$ is the boundary value of one in $H^{p}\left(\mathbb{C}_{ \pm}\right)$if and only if $H(f)=\mp i f$, where the Hilbert transform is taken to be the conventional branch; and, for the case $p=\infty$, the conventional Hilbert transform $\mathrm{Hf}$ is of type 1 or type 2, depending on $f \in H^{\infty}\left(\mathbb{C}_{+}\right)$or $f \in H^{\infty}\left(\mathbb{C}_{-}\right)$, respectively.

As an application of the main theorem, Theorem 1.2, we will establish in $\S 3$ the $L^{p}$-generalizations of the Bedrosian Theorem ([1]). There has been recent and growing interest in the Bedrosian identity due to its applications to adaptive decomposition of functions. The latter is a generalization of Fourier series expansion valid for functions defined in both compact and infinite intervals. The study projects crucial impacts on both theoretical and computational signal analysis. It is this trend

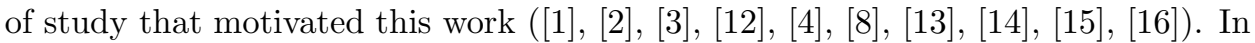
$\S 3$ we will include a short introduction to the main aspect of this study. We obtain the following:

Theorem 1.7. Suppose $f \in L^{p}(\mathbb{R}), g \in L^{q}(\mathbb{R}), 1 / p+1 / q \leq 1,1 \leq p, q \leq \infty$. If for some $\sigma>0, \operatorname{supp} \hat{f} \subset[-\sigma, \sigma]$ and supp $\hat{g} \subset \mathbb{R} \backslash[-\sigma, \sigma]$, then

$$
H(f g)=f H g,
$$

where the Hilbert transforms are taken to be the conventional branches.

We also have

Theorem 1.8. Suppose $f \in L^{p}(\mathbb{R})$ and $g \in L^{q}(\mathbb{R}), 1 / p+1 / q \leq 1,1 \leq p, q \leq \infty$. If supp $\hat{f} \subseteq[0, \infty)$, supp $\hat{g} \subseteq[0, \infty)$, then

$$
H(f g)=f H g,
$$


where the Hilbert transforms are taken to be the conventional branches and of type 1, if applicable.

And

Theorem 1.9. Suppose $f \in L^{p}(\mathbb{R})$ and $g \in L^{q}(\mathbb{R}), 1 / p+1 / q \leq 1,1 \leq p, q \leq \infty$. If $\operatorname{supp} \hat{f} \subseteq(-\infty, 0]$, supp $\hat{g} \subseteq(-\infty, 0]$, then

$$
H(f g)=f H g,
$$

where the Hilbert transforms are taken to be the conventional branches and of type 2, if applicable.

The proof of Theorem 1.7 with the shifting factor $e^{i 2 \pi \alpha x}=1$ offers the second and third versions of the Bedrosian theorem for $L^{p}$ as cited in Theorem 1.8 and Theorem 1.9. In fact, it is these simple cases that motivate the Hardy space study and the generalizations.

Thanks are due to Hans G. Feichtinger for beneficial discussions based on an earlier manuscript of the paper. In particular, his question motivated us to reformulate the results generalizing the Bedrosian Theorem through introducing the conventional Hilbert transformations.

\section{Proof of Theorem 1.2}

The main effort of the proof lies in the boundary cases $p=1$ and $p=\infty$. Since the distribution setting involves dual spaces and the dual space of $H^{1}$ is BMO, the proof is technically dependent on some fundamental developments of harmonic analysis in [5] and [18.

We will frequently refer to Plemelj's formula $\left([\underline{6})\right.$ : For $f \in L^{p}(\mathbb{R}), 1 \leq p<\infty$, and its Cauchy integral

$$
F(x+i y)=\frac{1}{2 \pi i} \int_{-\infty}^{\infty} \frac{f(t)}{t-(x+i y)}, \quad \pm y>0,
$$

there holds the relation

$$
\lim _{ \pm y \rightarrow 0+} F(x+i y)= \pm \frac{1}{2} f(x)+i \frac{1}{2} H f(x), \quad \text { a.e. } x .
$$

It is well known that if $f \in H^{p}\left(\mathbb{C}_{ \pm}\right), 1 \leq p<\infty$, then $f$ is given by the above Cauchy integral.

We will make use of the modified Hardy spaces $\tilde{H}_{ \pm}^{p}(\mathbb{R}), 1 \leq p \leq \infty$, defined as $\tilde{H}_{ \pm}^{p}(\mathbb{R}):=\left\{g\right.$ : There exists a complex-valued $f \in L^{p}(\mathbb{R})$ such that $\left.g=f \pm i H f\right\}$.

Due to Plemelj's formula and the $L^{p}(\mathbb{R})$ boundedness of Hilbert transformation for $1<p<\infty$, the spaces $\tilde{H}_{ \pm}^{p}(\mathbb{R})$ coincide with $H_{ \pm}^{p}(\mathbb{R})$ in the corresponding range of $p$. For $p=1$ and $p=\infty$, the respective $\tilde{H}_{ \pm}^{p}(\mathbb{R})$ are truly larger than the corresponding $H_{ \pm}^{p}(\mathbb{R})$. Indeed, for instance, $\tilde{H}_{+}^{1}(\mathbb{R})$ is the boundary value of a Cauchy integral with boundary data in $L^{1}(\mathbb{R})$, while $H_{+}^{1}(\mathbb{R})$ is the boundary value of a Cauchy integral with boundary data in $H_{+}^{1}(\mathbb{R})$. Since $H_{+}^{1}(\mathbb{R})$ is a proper subspace of $L^{1}(\mathbb{R})$, we conclude that $H_{+}^{1}(\mathbb{R})$ is a proper subspace of $\tilde{H}_{+}^{1}(\mathbb{R})$.

For the case $p=\infty$, if $f \in L^{\infty}(\mathbb{R})$, then $H f$ is in $\operatorname{BMO}(\mathbb{R})$ (see, for instance, [5]); while, if $f$ is in $H^{\infty}\left(\mathbb{C}_{+}\right)$, then both its boundary value $f$ and its Hilbert transform $H f$ are in $L^{\infty}(\mathbb{R})$. So, $H_{+}^{\infty}(\mathbb{R})$ is a proper subspace of $\tilde{H}_{+}^{\infty}(\mathbb{R})$. Below we will write 
$H_{+}^{p}(\mathbb{R})$ as $H^{p}(\mathbb{R})$ and $\tilde{H}_{+}^{p}(\mathbb{R})$ as $\tilde{H}^{p}(\mathbb{R})$ for simplicity, and sometimes even drop $\mathbb{R}$. Theory for $H_{-}^{p}(\mathbb{R})$ and $\tilde{H}_{-}^{p}(\mathbb{R})$ is similar.

Below we first extend Theorem 1.1 to the modified Hardy space.

Theorem 2.1. If $f$ is the tempered distribution arising from a function in $\tilde{H}^{p}(\mathbb{R})$, $1 \leq p \leq \infty$, then

$$
\langle\hat{f}, \phi\rangle=0, \text { for all } \phi \in \Phi^{-} .
$$

We will make use of Calderón-Zygmund decomposition (see page 17 of [18]). For $\lambda>0$, the Calderón-Zygmund decomposition of $f \in L^{1}(\mathbb{R})$ at the height $\lambda$ exhibits

$$
f=g+b=g+\sum_{k} b_{k},
$$

where

(i) $\|g\|_{2}^{2} \leq C \lambda\|f\|_{L^{1}}$

(ii) there exists a sequence of pairwise disjoint dyadic intervals $I_{k}$ so that the support of each $b_{k}$ is contained in $I_{k}$,

$$
\int_{\mathbb{R}} b_{k}(t) d t=0 \text { and } \int_{\mathbb{R}}\left|b_{k}(t)\right| d t \leq C \lambda\left|I_{k}\right|
$$

(iii) $\sum_{k}\left|I_{k}\right| \leq C \lambda^{-1}\|f\|_{L^{1}}$.

The Calderón-Zygmund decomposition leads to the following result.

Lemma 2.2. Let $\mathbb{S}$ denote the Schwartz class on $\mathbb{R}$. If $\phi \in \mathbb{S}$ and $f \in L^{1}(\mathbb{R})$, then

$$
\int_{\mathbb{R}}(H f)(t) \phi(t) d t=-\int_{\mathbb{R}} f(t)(H \phi)(t) d t .
$$

Proof. The Calderón-Zygmund decomposition of $f \in L^{1}(\mathbb{R})$ has the expansion (2.1). Since the function $g$ in (2.1) is in $L^{2}(\mathbb{R})$, the "anti-Hermitian" character of the Hilbert transform gives

$$
\int_{\mathbb{R}}(H g)(t) \phi(t) d t=-\int_{\mathbb{R}} g(t)(H \phi)(t) d t, \quad \forall \phi \in \mathbb{S},
$$

by the Fourier transform.

For the function $b$ in (2.1), we observe that it belongs to the Hardy space $H^{p}(\mathbb{R})$ for $1 / 2<p<1$. Owing to (ii) and (iii), this is obvious from the decomposition of $b$ into a sum of the $H^{p}$ atoms (see Chapter 3 of [18),

$$
b(t)=\sum_{k} b_{k}(t)=\sum_{k} \mu_{k} a_{k}(t),
$$

where $\mu_{k}=\lambda\left|I_{k}\right|^{1 / p}, a_{k}=\left[\lambda\left|I_{k}\right|^{1 / p}\right]^{-1} b_{k}$, and $\left\{a_{k}\right\}$ is a collection of $H^{p}$ atoms satisfying $\sum\left|\mu_{k}\right|^{p} \leq C \lambda^{p-1}\|f\|_{L^{1}}$.

Since the dual of $H^{p}$ is the space $\Lambda_{\alpha}(\mathbb{R})$ for $\alpha=p^{-1}-1$, and both $\phi$ and $H \phi$ are in $\Lambda_{\alpha}(\mathbb{R})$, the duality argument shows that

$$
\int_{\mathbb{R}}(H b)(t) \phi(t) d t=-\int_{\mathbb{R}} b(t)(H \phi)(t) d t, \quad \forall \phi \in \mathbb{S} .
$$

This, combined with (2.3), implies the identity (2.2). 
Proof of Theorem 2.1. We only need to prove the cases $p=1$ and $p=\infty$, as the cases $1<p<\infty$ are already included in Theorem 1.1.

For the case $p=1$, let $f+i H f$ belong to $\tilde{H}^{1}$ with $f \in L^{1}$. There exists a sequence of functions $f_{n} \in L^{1} \cap L^{2}$ such that $f_{n} \rightarrow f$ in the $L^{1}$ sense. This implies that $f_{n}+i H f_{n}$ converges to $f+i H f$ in the weak- $L^{1}$ sense. Since $\hat{\phi} \in L^{\infty}$, it follows from Lemma 2.2 that

$$
\lim _{n \rightarrow \infty} \int\left(H f_{n}\right)(t) \hat{\phi}(t) d t=\int(H f)(t) \hat{\phi}(t) d t .
$$

Consequently, we have that if $\phi \in \Phi^{-}$, then

$$
\lim _{n \rightarrow \infty} \int\left(f_{n}(t)+i\left(H f_{n}\right)(t)\right) \hat{\phi}(t) d t=\int(f(t)+i(H f)(t)) \hat{\phi}(t) d t
$$

As a consequence of Theorem 1.1 for $p=2$, the left-hand side of (2.5) for each $n$ is zero; hence the integral in the right-hand side also has zero value. This proves the assertion for $p=1$.

Next we consider the case $p=\infty$. Let $f \in L^{\infty}$. We need to show that for any $\phi \in \Phi^{-}$,

$$
\int_{\mathbb{R}}(f(t)+i(H f)(t)) \hat{\phi}(t) d t=0 .
$$

We first note that $f+i H f$ is in BMO and $\hat{\phi}$ is in $H_{+}^{1}$ and therefore the left-hand side of (2.6) in the functional sense is well-defined. Since for $f \in L^{\infty}$ and $\hat{\phi} \in H^{1}$ there holds

$$
\int_{\mathbb{R}}(H f)(t) \hat{\phi}(t) d x=-\int_{\mathbb{R}} f(t)(H \hat{\phi})(t) d t
$$

(see page 146 of [5]), we have that

$$
\int_{\mathbb{R}}(f(t)+i(H f)(t)) \hat{\phi}(t) d t=\int_{\mathbb{R}} f(t)((I-i H) \hat{\phi})(t) d t .
$$

The operator $I-i H$, however, annihilates functions in $H^{1}$. This concludes the theorem for $p=\infty$. The proof is complete.

Proof of Theorem 1.2. Write $f=f^{+}+f^{-}$, where $f^{ \pm}=(1 / 2)(f \pm i H f) \in \tilde{H}^{p}\left(\mathbb{C}_{ \pm}\right)$. Now we show $f^{-}=0$. For any $\varphi \in C_{0}^{\infty}$ one can write $\varphi=\varphi^{+}+\varphi^{0}+\varphi^{-}$, where $\varphi^{ \pm} \in \Phi^{ \pm}$and $\varphi^{0} \in C_{0}^{\infty}$. Thus,

$$
\begin{aligned}
\left\langle\hat{f^{-}}, \varphi\right\rangle & =\left\langle\hat{f^{-}}, \varphi^{+}+\varphi^{0}+\varphi^{-}\right\rangle \\
& =\left\langle\hat{f^{-}}, \varphi^{0}+\varphi^{-}\right\rangle \quad \text { (Theorem 2.1) } \\
& =\left\langle\hat{f^{-}}, \varphi^{0}\right\rangle+\left\langle\hat{f}-\hat{f^{+}}, \varphi^{-}\right\rangle \\
& =\left\langle\hat{f^{-}}, \varphi^{0}\right\rangle+\left\langle\hat{f}, \varphi^{-}\right\rangle-\left\langle\hat{f^{+}}, \varphi^{-}\right\rangle \\
& =\left\langle\hat{f^{-}}, \varphi^{0}\right\rangle, \quad \text { (by assumption and Theorem 2.1). }
\end{aligned}
$$

This shows that $\hat{f^{-}}$is supported at the single point zero. Hence (see, for instance, [7, 4.5, Chapter II),

$$
\hat{f^{-}}=\sum_{k=0}^{N} c_{k}\left(\frac{d}{d x}\right)^{k} \delta .
$$


Therefore, $f^{-}=\sum_{k=0}^{N} c_{k}(i x)^{k}$. This implies that $f^{-}=0$ for $1 \leq p<\infty$ and $f^{-}=c_{0}$ for $p=\infty$. In the latter case we obtain

$$
f=f^{+}+c_{0} \text {. }
$$

Indeed, the decomposition $f=f^{+}+f^{-}$in the case $p=\infty$ is unique modulo constants.

Now, the assumption $f \in L^{p}$ implies $f^{+}=f \in L^{p}$ and, in particular, $H f \in L^{p}$. Since $f=(1 / 2)(f+i H f)$, we have $H f=-i f$. Theorem [1.6] then implies that $f \in H^{p}$, where $1 \leq p \leq \infty$.

\section{Generalizations of the Bedrosian theorem}

The Bedrosian theorem was originally presented in [1] in the following form: Let $f, g$ be complex-valued signals in $L^{2}(\mathbb{R})$ of a real variable. If $\hat{f}(\omega)$ vanishes for $|\omega| \geq a$ and $\hat{g}(\omega)$ vanishes for $|\omega| \leq a$, where $a$ is a positive number, or, alternatively, if both $f, g$ are analytic singles in the sense that supp $\hat{f} \subseteq[0, \infty)$ and supp $\hat{g} \subseteq[0, \infty)$, then the Hilbert transform of the product of $f$ and $g$ fulfills the relation

$$
H[f g]=f H g .
$$

Below we include an introduction to adaptive decomposition of functions and its relation to the Bedrosian identity.

In relation to the engineering development of the so called EMD algorithm (3), there has been increasing interest in adaptive decomposition of functions into the form

$$
f(t)=\sum_{k=1}^{\infty} \rho_{k}(t) \cos \theta_{k}(t)
$$

where $\rho_{k} \geq 0$ a.e., $\theta_{k}^{\prime}(t) \geq 0$ a.e., and $\rho_{k}(t) \cos \theta_{k}(t)$ satisfies the Hilbert transform relation

$$
H\left(\rho_{k} \cos \theta_{k}\right)(t)=\rho_{k}(t) \sin \theta_{k}(t) \quad \text { a.e. }
$$

Each basic entry $\rho_{k}(t) \cos \theta_{k}(t)$ that enjoys the three properties listed above is called a mono-component ([16]). The non-negative requirement for $\rho_{k}$ is basic, as it is the amplitude of the entry. The non-negative requirement for the derivative function $\theta_{k}^{\prime}$ amounts to requiring that each entry has non-negative frequency, in accordance with its physics meaning. It is also called instantaneous frequency or analytic instantaneous frequency by signal analysts. The final Hilbert transform relation requires that each entry is the boundary value of an analytic function in the upper-half complex plane. This is called, in physics, a physical realizable signal. Fourier series form a particular case of this type of series, as each entry of $a_{n} \cos n t$ and $b_{n} \sin n t$ is a mono-component: Each entry of these may be written in the amplitude-frequency form that enjoys the three properties. "Adaptive" in our formulation means fastest convergence in a certain metric space. Adaptive decomposition of this type has very good localization properties and is considered to be ideal for signal analysis. The relevant literature may be found in [1, 2], 3], [12, 4, [8, 13, 14, 15, 16] and their references. To achieve the goal of adaptive decomposition we need to find a large pool of mono-components. It has been known that the real parts of the boundary values of all starlike and $p$-starlike functions are mono-components. One of the ways to construct mono-components is to start from 
a unimodular mono-component, of the form $\cos \theta(t)$, where the function $\theta$ satisfies the condition $\theta^{\prime}(t) \geq 0$ a.e. and the relation

$$
H(\cos \theta)(t)=\sin \theta(t) .
$$

Examples of unimodular mono-components of this type include the real parts of the boundary values of Möbius transforms, further extending to finite Blaschke products (13, 14, 15]). Now suppose that the Bedrosian Theorem may be extended to the $L^{p}$ cases and that $\rho(t)$ and $\cos \theta(t)$ satisfy the required assumptions; then

$$
H(\rho \cos \theta)(t)=\rho(t) H(\cos \theta)(t)=\rho(t) \sin \theta(t) .
$$

Based on this procedure, and other generalizations of the Bedrosian Theorem as well, a large number of mono-components have been constructed ([20]).

So far all studies of the Bedrosian identity have been based on the $L^{2}$ formulation. In engineering practice the identity is applied to the cases where $f \in L^{p}(\mathbb{R})$ and $g \in L^{\infty}(\mathbb{R})$ without any mathematical justification. This gives an impetus to study $L^{p}$ generalizations of Bedrosian identity.

Now we turn to proving our $L^{p}$ generalizations of the Bedrosian Theorem. We first extend the translation property of the Fourier transform for $L^{1}(\mathbb{R})$ to tempered distributions. In the following theorem we denote the function $e^{-2 \pi i a t}$ by $e^{-2 \pi i a(\cdot)}, t \in \mathbb{R}$.

Theorem 3.1. Let $T$ be a tempered distribution and $\operatorname{supp} \hat{T} \subset(A, B)$, where $A, B$ are in the generalized real number system. Then

$$
\operatorname{supp}\left(T e^{-2 \pi i \sigma(\cdot)}\right)^{\wedge} \subset(A+\sigma, B+\sigma) .
$$

Proof. Let $\varphi \in C_{0}^{\infty}$ be a test function; then

$$
\begin{aligned}
\left\langle\left(T e^{-2 \pi i \sigma(\cdot)}\right)^{\wedge}, \varphi\right\rangle & =\left\langle T, e^{-2 \pi i \sigma(\cdot)} \hat{\varphi}\right\rangle \\
& =\left\langle T,[\varphi(\cdot-\sigma)]^{\wedge}\right\rangle \\
& =\langle\hat{T}, \varphi(\cdot-\sigma)\rangle .
\end{aligned}
$$

Since supp $\varphi(\cdot-\sigma) \subset \mathbb{R} \backslash(A, B)$ if and only if supp $\varphi \subset \mathbb{R} \backslash(A+\sigma, B+\sigma)$, we conclude the theorem.

We are now ready to give

Proof of Theorem 1.7. We first decompose $g$ into $g=g^{+}+g^{-}$so that supp $\hat{g^{+}} \subset$ $[\beta, \infty)$ and supp $\hat{g^{-}} \subset(-\infty,-\beta]$, where $\beta>\sigma$. If we can show

$$
H\left(f g^{ \pm}\right)=f H\left(g^{ \pm}\right),
$$

then the linearity of Hilbert transformation will imply that $H(f g)=f H g$. We only prove the case for $g^{+}$, as the case for $g^{-}$is similar. Write $f g^{+}=\left(f e^{-2 \pi i \alpha x}\right)$ $\cdot\left(e^{2 \pi i \alpha x} g^{+}\right)$for any $\alpha$ in between $\sigma$ and $\beta$. Since supp $\hat{f} \subset[-\sigma, \sigma]$, from Theorem 3.1 we have supp $\left(f e^{-2 \pi i \alpha \cdot}\right)^{\wedge} \subset[0,+\infty)$. By invoking Theorem [1.2, $f e^{-2 \pi i \alpha x}$ is the boundary value of a function in $H^{p}\left(\mathbb{C}_{+}\right)$. Since supp $\hat{g}^{+} \subset[\beta, \infty)$, from Theorem 3.1. we have $\operatorname{supp}\left(e^{2 \pi i \alpha \cdot} g^{+}\right)^{\wedge} \subset[0,+\infty)$. Invoking Theorem 1.2 again, $e^{2 \pi i \alpha x} g^{+}$is the boundary value of a function in $H^{q}\left(\mathbb{C}_{+}\right)$. When $1<p, q<\infty$ with 
$1 / p+1 / q \leq 1$, we have, for $1 \leq r<\infty, 1 / r=1 / p+1 / q$,

$$
\begin{gathered}
\sup _{0<y<\infty} \int_{\mathbb{R}}\left|f(x+i y) e^{-2 \pi i \alpha(x+i y)}\right|^{r}\left|e^{2 \pi i \alpha(x+i y)} g^{+}(x+i y)\right|^{r} d x \\
\leq \sup _{0<y<\infty}\left(\int_{\mathbb{R}}\left(\left|f(x+i y) e^{-2 \pi i \alpha(x+i y)}\right|^{r}\right)^{\frac{p}{r}} d x\right)^{\frac{r}{p}} \\
\cdot\left(\int_{\mathbb{R}}\left(\left|e^{2 \pi i \alpha(x+i y)} g^{+}(x+i y)\right|^{r}\right)^{\frac{q}{r}} d x\right)^{\frac{r}{q}} \\
\leq \| f(\cdot) e^{-2 \pi i \alpha^{*} \cdot\left\|_{H^{p}}^{r}\right\| e^{2 \pi i \alpha \cdot} g^{+}(\cdot) \|_{H^{q}}^{r}<\infty .}
\end{gathered}
$$

Therefore, $f(x) g^{+}(x)=\left(f(x) e^{-2 \pi i \alpha x}\right)\left(e^{2 \pi i \alpha x} g^{+}(x)\right)$ is the boundary value of a function in $H^{r}\left(\mathbb{C}_{+}\right)$. For $p=\infty$ or $p=1$, it is obvious that $f g^{+}$is the boundary value of a function in $H^{q}\left(\mathbb{C}_{+}\right)$or $H^{p}\left(\mathbb{C}_{+}\right)$, respectively. In all cases, by virtue of Theorem 1.6 we have

$$
H\left(f g^{+}\right)=-i\left(f g^{+}\right)=f\left(-i g^{+}\right)=f H g^{+},
$$

proving the Bedrosian identity for this case. The proof is complete.

We note that Fourier transforms of the functions in Theorem 1.7 in the $q=\infty$ case do not involve non-trivial multiples of the Dirac function; thus there is no need to specify whether it is the type- 1 or type- 2 conventional Hilbert transform that is in use.

Finally we give a remark to end the paper.

Remark 3.2. The literature [1] cites three examples for the classic Bedrosian Theorem:

(i) For $n=0,1, \ldots$, let $J_{n}$ denote the Bessel functions. Then, for $0<b<a$,

$$
H\left[\sin a x J_{n}(b x)\right]=\cos a x J_{n}(b x) .
$$

(ii) Let

$$
C i(a x):=-\int_{a x}^{\infty} \frac{\cos t}{t} d t, \quad s i(x):=-\int_{x}^{\infty} \frac{\sin t}{t} d t .
$$

It is known that

$$
H\left(C_{i}(a x)\right)=(-\operatorname{sgn} x) \operatorname{si}(a|x|) .
$$

Then, for $0<b<a$,

$$
H[\sin b x C i(a x)]=\sin b x(-\operatorname{sgn} x) \operatorname{si}(a|x|) .
$$

(iii) Assume that the highest frequency component in $r(t)$ is less than $\omega_{0}$ and $\omega_{0}$ and $\phi$ are constants. Then,

$$
H\left[r(t) \cos \left(\omega_{0} t+\phi\right)\right]=r(t) \sin \left(\omega_{0} t+\phi\right), \quad \omega_{0}>0 .
$$

We note that in all the above examples it is the generalized Bedrosian Theorem 1.7 for $L^{p}$ spaces that should be used.

\section{REFERENCES}

1. E. Bedrosian, A product theorem for Hilbert transform, Proc. IEEE, 51 (1963), 868-869.

2. J. L. Brown, Jr., A Hilbert transform product theorem, Proc. IEEE, 74 (1986), 520-521.

3. Q. Chen, N. Huang, S. Riemenschneider and Y. Xu, A B-spline approach for empirical mode decompositions, Adv. Comput. Math., 24 (2006), 171-195. MR2222267(2007e:94010)

4. L. Cohen, Time-Frequency Analysis: Theory and Application, Prentice Hall, Englewood Cliffs, NJ, 1995. 
5. C. Fefferman and E.M. Stein, $H^{p}$ spaces of several variables, Acta Math., 129 (1972), 137-195. MR 0447953 (56:6263)

6. J. B. Garnett, Bounded Analytic Functions, Academic Press, 1987. MR628971 (83g:30037)

7. I. M. Gel'fand and G. E. Shilov, Generalized Functions, Vol. 2, Academic Press Inc., 1968. MR 0230128 (37:5693)

8. N. Huang et al., The empirical mode decomposition and the Hilbert spectrum for nonlinear and non-stationary time series analysis, Proc. R. Soc. London, 454 A (1998), 903-995. MR1631591 (99d:76082)

9. B. H. Li, On distributions with parameter and their analytic representations, Chinese Math. Ann., 2(4) (1981), 399-405. MR670677 (83k:46037)

10. B.H. Li and L.K. Guo, Riesz transformations of distributions and a generalized Hardy space, Approx. Theory and its Appl., 5(4) (1988), 1-17. MR.1055661 (91h:46077)

11. J. N. Pandey, The Hilbert Transform of Schwartz Distributions and Applications, WileyInterscience, 1996. MR.1363489 (97e:44010)

12. B. Picinbono, On instantaneous amplitude and phase of signals, IEEE Transactions on Signal Processing, vol. 45, No. 3, March 1997, 552-560.

13. T. Qian, Q-H. Chen and L-Q. Li, Analytic unit quadrature signals with non-linear phase, Physica D: Nonlinear Phenomena, 203 (2005), 80-87. MR2135134(2006k:94025)

14. T. Qian, Analytic signals and harmonic measures, J. Math. Anal. Appl., 314 (2006), 526-536. MR 2185248 (2006k:94026)

15. T. Qian, Characterization of boundary values of functions in Hardy spaces with applications in signal analysis, J. Integral Equations Appl., Vol. 17, No. 2, Summer 2005, 159-198. MR 2182189 (2006g:30058)

16. T. Qian, Mono-components for decomposition of signals, Math. Meth. Appl. Sci., 29 (2006), 1187-1198. MR2237711 (2007e:44008)

17. W. Rudin, Real and Complex Analysis, McGraw-Hill, 1966. MR0210528 (35:1420)

18. E. M. Stein, Harmonic Analysis: Real Variable Methods, Orthogonality and Oscillatory Integrals, Princeton Univ. Press, Princeton, NJ (1993). MR1232192 (95c:42002)

19. Y. Xu and D. Yan, The Bedrosian identity for the Hilbert transform of product functions, Proc. Amer. Math. Soc., 134 (2006), 2719-2728. MR2213752(2008b:65176)

20. B. Yu and H-Z. Zhang, On the Bedrosian identity, preprint (accepted).

Department of Mathematics, Faculty of Science and Technology, University of Macau, Macao, China SAR

E-mail address: fsttq@umac.mo

Department of Mathematics, Syracuse University, Syracuse, New York 12344

E-mail address: yxu06@syr.edu

School of Information Science and Engineering, The Graduate University of the Chinese Academy of Sciences, Beijing 100080, People's Republic of China

E-mail address: ydunyan@gucas.ac.cn

Department of Mathematics, Zhongshan University, Guangzhou 510275, People's Republic of China

E-mail address: mcsylx@mail.sysu.edu.cn

Academy of Mathematics and Systems Science, Chinese Academy of Sciences, Beijing, 100080, People's Republic of China

E-mail address: yubo2003@amss.ac.cn 